\title{
A comparison between Poisson and zero-inflated Poisson regression models with an application to number of black spots in Corriedale sheep
}

\author{
Hugo NAYA ${ }^{1,2,3 *}$, Jorge I. Urioste ${ }^{2}$, Yu-Mei $\mathrm{CHANG}^{3}$, \\ Mariana Rodrigues-Motta ${ }^{3}$, Roberto Kremer ${ }^{4}$, Daniel Gianola ${ }^{3}$ \\ ${ }^{1}$ Unidad de Bioinformática, Institut Pasteur de Montevideo, Mataojo 2020, \\ Montevideo 11400, Uruguay \\ ${ }^{2}$ Departamento de Producción Animal y Pasturas, Facultad de Agronomía, Av. Garzón 780, \\ Montevideo 12900, Uruguay \\ ${ }^{3}$ Department of Animal Sciences, University of Wisconsin-Madison, Madison, \\ WI 53706, USA \\ ${ }^{4}$ Departamento de Ovinos y Lanas, Facultad de Veterinaria, Av. Lasplaces 1550, \\ Montevideo 11600, Uruguay
}

(Received 15 October 2007; accepted 16 January 2008)

\begin{abstract}
Dark spots in the fleece area are often associated with dark fibres in wool, which limits its competitiveness with other textile fibres. Field data from a sheep experiment in Uruguay revealed an excess number of zeros for dark spots. We compared the performance of four Poisson and zero-inflated Poisson (ZIP) models under four simulation scenarios. All models performed reasonably well under the same scenario for which the data were simulated. The deviance information criterion favoured a Poisson model with residual, while the ZIP model with a residual gave estimates closer to their true values under all simulation scenarios. Both Poisson and ZIP models with an error term at the regression level performed better than their counterparts without such an error. Field data from Corriedale sheep were analysed with Poisson and ZIP models with residuals. Parameter estimates were similar for both models. Although the posterior distribution of the sire variance was skewed due to a small number of rams in the dataset, the median of this variance suggested a scope for genetic selection. The main environmental factor was the age of the sheep at shearing. In summary, age related processes seem to drive the number of dark spots in this breed of sheep.
\end{abstract}

zero-inflated Poisson / sheep / spot / posterior predictive ability / Bayesian hierarchical model

*Corresponding author: naya@pasteur.edu.uy 


\section{INTRODUCTION}

The presence of black-brown fibres in wool from Corriedale sheep is recognised as a fault $[13,20]$. This issue limits the competitiveness of wool with other textile fibres and reduces its value by $15-18 \%$ when the number exceeds 300 fibres $\cdot \mathrm{kg}^{-1}$ top (Frank Racket, 1997, personal communication). In Uruguayan wool, this value can be as large as 5000 fibres $\mathrm{kg}^{-1}$ top, with most of the dark fibres having an environmental origin, e.g. faeces and urine dyeing $[3,18]$. With appropriate clip preparation, values ranging from 800 to 1000 fibres have been found, and these probably have a genetic background. Skin spots with blackbrown fibres and isolated pigmented fibres are the probable origin of these fibres $[2,9,12,20]$.

With the aim of investigating factors involved in the development of pigmented fibres, an experiment was carried out in which fleeces of animals from two experimental flocks were sampled yearly at shearing for laboratory analysis. Each animal was inspected, and the number of black spots, their diameter and the estimated percentage of dark fibres in each spot were recorded. While genetic selection should focus on reducing the number of dark fibres, it is expensive and cumbersome to record such a value for each animal on a routine basis. Laboratory techniques are labour intensive and slow.

In this context, the number of dark spots in the fleece area of animals may be a useful indicator trait, for several reasons. First, our empirical observations suggest that dark fibres are associated with dark spots, hinting a positive correlation between the two variables. Second, spots can be assessed easily and quickly, and scoring is less subjective than for other candidate measures such as the percentage of spot area with dark fibres $[1,10,11]$. Third, we have observed that in spots without or with dark fibres in young animals, the presence of dark fibres increases with age. Hence, the presence of spots indicates dark fibres in adult animals. If laboratory analyses confirm that black spots are positively correlated with the number of dark fibres, recording on a nation-wide basis would be straightforward.

Previous studies in Romney sheep [6] have addressed the occurrence of black wool spots at weaning $\left(\mathrm{BWS}_{\mathrm{w}}\right)$ and at yearling age $\left(\mathrm{BWS}_{\mathrm{y}}\right)$. Enns and Nicoll [6] used a threshold model for a binary response variable (the presence or absence of pigmented spots), and their largest heritability estimates were $0.070(0.018)$ and $0.072(0.014)$ for $\mathrm{BWS}_{\mathrm{w}}$ and $\mathrm{BWS}_{\mathrm{y}}$, respectively. In contrast, in our research, focus has been on modelling the number of dark spots in each animal, irrespective of the presence of dark fibres. As a count variable, the number of spots could plausibly follow a Poisson distribution. However, as shown in Figure 1, there is an excess of zeros in the empirical distribution for field records, 


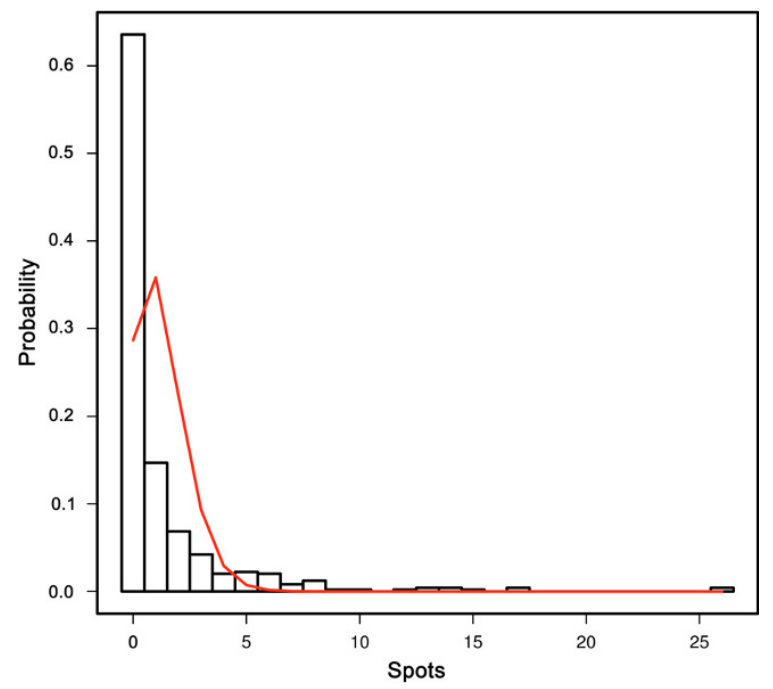

Figure 1. Distribution of the number of black spots in field data $(n=497)$. The solid line represents the best fit of a Poisson distribution to the observed data, fitted with package "gnlm" (http://popgen.unimaas.nl/ jlindsey/rcode.html) of $R$ [26].

relative to their expected value under Poisson sampling with a homogeneous parameter. If $Y$ follows a Poisson distribution, then $E(Y)=\operatorname{Var}(Y)$, where $E(\cdot)$ and $\operatorname{Var}(\cdot)$ represent the mean and variance, respectively. In a Poisson distribution, the variance-to-mean-ratio (VTMR) is 1 . In the observed data in Figure 1, VTMR was 6.8. A zero-inflated Poisson model (ZIP) [17], may provide a better description of the data. This model assumes that observations come from one of two different components, a "perfect" state which produces only zeros with probability $\theta$, and an "imperfect" one that follows a Poisson distribution, with probability $(1-\theta)$ and Poisson parameter $\lambda$. It can be shown that the mean and variance of a ZIP variate are

$$
E(Y)=(1-\theta) \lambda
$$

and

$$
\operatorname{Var}(Y)=E(Y)(1+\theta \lambda)
$$

respectively, which accounts for VTMR $>1$ provided that overdispersion arises from an excess of zeros. Zero-inflated models for count data in animal breeding have been discussed by Gianola [15] and used by Rodrigues-Motta and collaborators [27] in an analysis of the number of mastitis cases in dairy cattle. 
From previous exploratory analysis [16,24], the age of animals appears to be a main source of variability of the number of spots, with flock and year having marginal effects. Modelling can proceed along the lines of generalised linear models [21] or generalised linear mixed models [25] provided that the link function used is appropriate. However when mixture distributions are assumed, as in the ZIP model, estimation is more involved, since indicator variables (e.g., from which of the two states a zero originates) are not observed. However, imputation of non-observed parameters given the data fits naturally in the Bayesian framework [19]. In recent years, Bayesian Markov chain Monte Carlo (MCMC) methods have become widely used in animal breeding [28], as a powerful and flexible tool. An advantage of the Bayesian MCMC framework is that it is relatively easy to implement measures of model quality such as posterior predictive ability (PPA) checks.

In this paper, four different candidate models for the number of spots were compared. Poisson and ZIP models were considered, with the log of the Poisson parameter of each of the models regressed on environmental and genetic effects. The two models were extended further to include a random residual in the regression, aimed to capture overdispersion other than that due to extra zeros. Two of the models were selected and fitted to a sample of Corriedale sheep to obtain estimates of population parameters.

\section{MATERIALS AND METHODS}

\subsection{Simulation}

Four different scenarios $(\mathrm{H} 1-\mathrm{H} 4)$ were simulated as described in Table I. The rationale underlying the models is that the observed number of spots in each animal follows a Poisson distribution with the logarithm of its parameter expressed as a linear model. The Poisson distribution does not accommodate well the overdispersion caused by excess zeros, so a ZIP model is a reasonable competitor. Furthermore, the parameter of the Poisson distribution represents the expected propensity of spots, so an additional error (residual) term at the regression level allows modelling individual differences in propensity. The two models (Poisson and ZIP), each with or without residuals, give the four models $(P, Z, P e$ and $Z e$ ) studied.

Data were generated from either ZIP (H1, H2) or Poisson (H3, H4) distributions; the log of the Poisson parameter contained (H2, H4) or did not contain $(\mathrm{H} 1, \mathrm{H} 3)$ a random residual. In all four models, the ram effects were assumed to follow independent normal distributions with null mean and variance $\sigma_{\mathrm{ram}}^{2}$; the residual was independent and identically distributed as $e_{i, j, k} \sim N\left(0, \sigma_{e}^{2}\right)$ (H2, H4). 
Table I. Model label, simulated data distribution given the parameters, regression function and name of each scenario $(\mathrm{H} 1, \mathrm{H} 2, \mathrm{H} 3, \mathrm{H} 4)$.

\begin{tabular}{cccc}
\hline Model & Distribution & Regression & Scenario \\
\hline$Z$ & $y_{i, j, k} \sim \operatorname{ZIP}\left(\theta, \lambda_{i, j}\right)$ & $\log \left(\lambda_{i, j}\right)=b_{0}+b_{1} \cdot \operatorname{age}_{i}+\operatorname{ram}_{j}$ & H1 \\
$Z e$ & $y_{i, j, k} \sim \operatorname{ZIP}\left(\theta, \lambda_{i, j, k}\right)$ & $\log \left(\lambda_{i, j, k}\right)=b_{0}+b_{1} \cdot \operatorname{age}_{i}+\operatorname{ram}_{j}+e_{i, j, k}$ & $\mathrm{H} 2$ \\
$P$ & $y_{i, j, k} \sim \operatorname{Poisson}\left(\lambda_{i, j}\right)$ & $\log \left(\lambda_{i, j}\right)=b_{0}+b_{1} \cdot \operatorname{age}_{i}+\operatorname{ram}_{j}$ & $\mathrm{H} 3$ \\
$P e$ & $y_{i, j, k} \sim \operatorname{Poisson}\left(\lambda_{i, j, k}\right)$ & $\log \left(\lambda_{i, j, k}\right)=b_{0}+b_{1} \cdot \operatorname{age}_{i}+\operatorname{ram}_{j}+e_{i, j, k}$ & $\mathrm{H} 4$ \\
\hline
\end{tabular}

The $b$ 's are unknown regressions.

In each scenario, 100 datasets (replicates) were randomly generated, with 1000 observations each. For each animal, the covariate age was randomly sampled, resembling the distribution of the age in the observed data. Forty rams (sires) were sampled in each dataset and randomly assigned to observations. In each scenario, the true parameters were selected to resemble the observed distribution of spots.

\subsection{Models fitted in the simulation}

Four models were fitted to the simulated data $(Z, Z e, P$ and $P e)$, each matching a specific scenario, as shown in Table I. Models are connected as illustrated in Figure 2. A path between two models involves fixing or adding a single parameter. Preliminary analysis indicated that flock and year effects (and their interaction) had minor importance, so these factors were not included in the simulations. However, when models were fitted to the real data, the regression models included flock and year effects.

\subsection{Bayesian computation}

Parameter inference was done using the OpenBUGS software [31]. Vague priors were assigned to represent initial uncertainty. A normal distribution centred at zero with precision 0.01 was used for location parameters, while a Gamma $(0.01,0.01)$ distribution was assumed for each of the two variance parameters. Several different hyper-parameter values were assigned in pilot runs, with the only observable difference being the time needed to attain convergence. For each scenario and model, the burn-in period was determined from preliminary runs, based on four chains, starting at different points. Final runs were performed with two chains each. The burn-in period was of 10000 iterations, and samples were obtained from the following 10000 iterations, without thinning. An exception was model $\mathrm{Pe}$ in scenario $\mathrm{H} 4$, where the required burn-in period was 30000 iterations. 


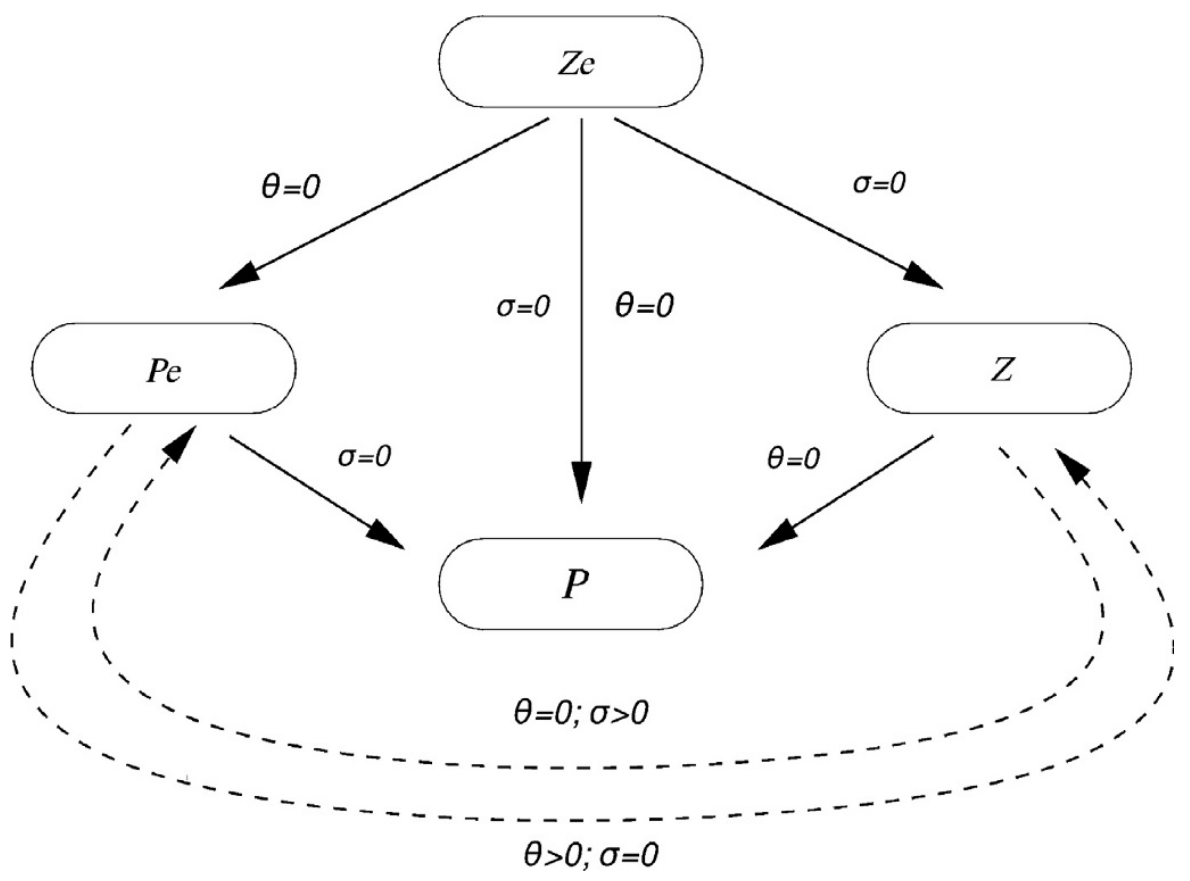

Figure 2. Graphical display of the four models considered. Distances depend on one or two parameters. $\theta$ is probability of the perfect state; $\sigma$ is the standard deviation of the error term in the regression. Dashed lines connect models that need to incorporate one parameter while fixing the other parameter to zero.

\subsection{End points for model comparison}

Models were contrasted first through simulated data (comparing true and estimated parameter values) and by using the deviance information criterion (DIC), estimates of marginal likelihoods with the method of Newton and Raftery [22] and via PPA. The DIC [30] was obtained directly from OpenBUGS. PPA was patterned after Sorensen and Waagepetersen [29]. Suppose that for a model $M, \theta_{M}^{(k)}, k=1, \ldots, K$, is drawn from the posterior distribution of the parameter vector $\theta_{M}$, and that, subsequently, replicate data $\mathbf{y}_{M}^{(k)}$ are generated given $\theta_{M}^{(k)}$ as true parameters. Given some univariate discrepancy statistic $T\left(\mathbf{y}, \theta_{M}\right)$, it is possible to study the predictive ability of model $M$ from samples drawn from the posterior distribution of the difference $T\left(\mathbf{y}, \theta_{M}^{(k)}\right)-T\left(\mathbf{y}_{\text {rep }}^{(k)}, \theta_{M}^{(k)}\right)$. For the Poisson model we used

$$
T\left(\mathbf{y}, \theta_{M}\right)=\sum_{k=1}^{K}\left(\frac{\mathbf{y}-\lambda_{k}}{\sqrt{\lambda_{k}}}\right)^{2}
$$


as discrepancy statistic, where $\lambda_{k}$ in the numerator is the mean, and $\sqrt{\lambda_{k}}$ in the denominator is the standard deviation; for the ZIP model, the mean and standard deviation were replaced by their corresponding values.

\subsection{Field data}

Records were collected in 2002-2004 from two experimental flocks belonging to the Universidad de la República, Uruguay. After edits, 497 records from sheep with known sire (ram) were kept; 37, 182 and 278 records were from 2002, 2003 and 2004, respectively; 407 and 90 were from flocks 1 and 2, respectively. Genetic connection was through two rams with progeny in both flocks; a total of 19 rams had progeny. In our dataset 36 animals had records in both 2002-2003, 71 in 2003-2004 and 27 animals had measures in all three years. For simplicity, dependence between observations from the same animal was ignored, so that the only source of correlation considered was that resulting from a half-sib family structure. Clearly, the limited dataset precludes precise estimation of genetic parameters, but this was not an objective of this study.

\section{RESULTS}

\subsection{Simulations}

For each scenario simulated, the results are presented for the "true" model and for the other three models. Values of the DIC (highlighting $p D$, the "effective number of parameters") and of the difference statistic used for PPA are shown in Table II.

\subsubsection{DIC}

In scenario H1, where $Z$ is the true model, $P$ e performed better (lower DIC) than the true model, in spite of the penalty resulting from a larger $p D$ (number of parameters). The value of nearly 400 effective parameters in 1000 observations indicates that very few observations clustered under the same Poisson distribution. Models with residuals had a higher $p D$ but lower deviance. Except for the $P$ model, the other specifications had similar DIC, at least in the light of the between replicates standard deviation. Clearly the Poisson model was the worst under the "true ZIP" scenario.

In scenario $\mathrm{H} 2$ ( $Z e$ is the true model), $P e$ was, again, better than the true model and the picture with respect to $p D$ was as in the H1 scenario, although differences between models with residuals were smaller. Models without residuals had the poorest performance; $P$ had the worse DIC. 
Table II. Averages and standard deviations of deviance information criterion (DIC), effective number of parameters $(p D)$ and difference statistic for the posterior predictive ability (DPPA) for the four models in each scenario over 100 replicates.

\begin{tabular}{ccrrrrrc}
\hline Scenario & Model & \multicolumn{1}{c}{ DIC } & \multicolumn{1}{c}{ s.d. } & \multicolumn{1}{c}{$p D$} & \multicolumn{1}{c}{ s.d. } & \multicolumn{1}{c}{ DPPA } & s.d. \\
\hline H1 & $\boldsymbol{Z}$ & 2368.0 & 101.8 & 17.3 & 1.3 & 0.003 & 0.030 \\
& $Z e$ & 2368.9 & 101.5 & 34.1 & 6.8 & -0.006 & 0.028 \\
& $P$ & 3138.5 & 196.6 & 17.8 & 0.8 & 1.204 & 0.137 \\
H2 & $P e$ & 2262.5 & 102.7 & 399.6 & 18.9 & 0.050 & 0.009 \\
& $Z$ & 2645.1 & 117.4 & 18.1 & 1.0 & 0.175 & 0.055 \\
& $\boldsymbol{Z e}$ & 2521.3 & 103.7 & 135.0 & 17.8 & 0.007 & 0.011 \\
& $P$ & 3579.9 & 222.3 & 18.2 & 0.6 & 1.878 & 0.239 \\
$\mathrm{H} 3$ & $P e$ & 2308.2 & 101.7 & 431.3 & 18.1 & 0.030 & 0.009 \\
& $Z$ & 1768.4 & 77.8 & 16.8 & 1.1 & -0.006 & 0.049 \\
& $Z e$ & 1769.9 & 77.5 & 31.9 & 6.3 & -0.021 & 0.050 \\
& $\boldsymbol{P}$ & 1766.6 & 77.9 & 16.7 & 1.0 & 0.007 & 0.052 \\
$\mathrm{H} 4$ & $P e$ & 1768.2 & 77.8 & 33.1 & 6.3 & -0.007 & 0.046 \\
& $Z$ & 1962.9 & 127.7 & 17.8 & 1.0 & 0.177 & 0.085 \\
& $Z e$ & 1848.2 & 84.3 & 125.1 & 20.1 & -0.006 & 0.033 \\
& $P$ & 1958.4 & 107.5 & 17.3 & 1.0 & 0.276 & 0.101 \\
& $\boldsymbol{P e}$ & 1842.7 & 84.1 & 131.8 & 19.0 & 0.007 & 0.034 \\
\hline
\end{tabular}

The model corresponding to each scenario is in boldface.

Table III. Times a given model was the best when selected by DIC (over 100 replicates).

\begin{tabular}{ccccr}
\hline Model & $Z$ & $Z e$ & $P$ & $P e$ \\
\hline H1 & 0.5 & 0.5 & 0.0 & $\mathbf{9 9}$ \\
H2 & 0 & 0 & 0 & $\mathbf{1 0 0}$ \\
H3 & 2 & 3.5 & $\mathbf{7 7 . 5}$ & 17 \\
H4 & 1 & 0 & 0 & $\mathbf{9 9}$ \\
\hline
\end{tabular}

Fractional numbers correspond to ties. The "true" models are in the diagonal while the "winner" model for each scenario is shown in boldface.

Under H3 ( $P$ is the true model) all DIC values were similar. Models with residuals had smaller $p D$ than in scenarios $\mathrm{H} 1, \mathrm{H} 2$, probably due to the simpler nature of this simulation scenario. Finally, in scenario H4, the true model $(\mathrm{Pe})$ was best under the DIC, followed by Ze.

The global picture is clearer when the number of times (in 100 simulations) in which each model had the smallest DIC was considered (Tab. III). Model Pe outperformed other models except under H3. Notably, DIC selected the right model only in 172 out of 400 comparisons ( $43 \%$ of the time). 
Table IV. Mean and standard deviation (in 100 runs) of the harmonic mean of sampled log-likelihoods for each model and scenario.

\begin{tabular}{ccccccccc}
\hline Model & $Z$ & s.d. & \multicolumn{1}{c}{$Z e$} & s.d. & $P$ & \multicolumn{1}{c}{ s.d. } & $P e$ & s.d. \\
\hline H1 & -1184.1 & 50.5 & -1180.2 & 50.6 & -1569.1 & 98.3 & -1007.7 & 44.8 \\
H2 & -1322.4 & 58.5 & -1235.7 & 51.0 & -1789.6 & 111.0 & -1012.5 & 46.0 \\
H3 & -884.8 & 38.6 & -881.4 & 38.5 & -883.9 & 38.8 & -880.2 & 38.8 \\
H4 & -974.5 & 53.0 & -902.8 & 40.0 & -979.4 & 53.7 & -898.9 & 40.3 \\
\hline
\end{tabular}

\subsubsection{PPA}

Values of the PPA difference statistic close to zero indicate essentially no differences between observed and predicted responses. In regard to the PPA results, the true model always predicted best, and this was essentially true for all scenarios (Tab. II). The pure Poisson model $(P)$ performed badly in ZIP scenarios (H1 and $\mathrm{H} 2$ ), while $\mathrm{Ze}$ did reasonably well in all four scenarios. In H3, PPA was similar for all models. The problem with this criterion seems to be its low discriminative power, relative to its high standard deviations over replications. Alternatives to PPA are cross-validation techniques, but these were not considered due to computational expense.

\subsubsection{Marginal likelihood}

It was impossible to calculate the Bayes factor for several pairs of models, given the huge differences in marginal likelihoods. For this reason, only estimates of marginal log-likelihood are presented for each model and scenario (Tab. IV). On the basis of this criterion, $P e$ was the best model in all scenarios.

\subsubsection{Parameter inference}

As expected, parameter estimates were in agreement with their "true" values when a model matched its corresponding scenario (Tab. V). However, when models pertained to a different scenario, their performances were markedly different. Regressions on age were well inferred, but estimated intercepts $b_{0}$ were severely understated when Poisson models were applied to ZIP scenarios. Model $Z e$ estimates were always in agreement with the "true" values, regardless of the scenario. $P e$ model estimates of intercept and of the residual variance were strongly biased in ZIP scenarios. Finally, models with residuals estimated the sire (ram) variance well.

The ability of different models to predict breeding values is of interest. Given that the "true" values of rams were known, their Spearman rank correlation with 

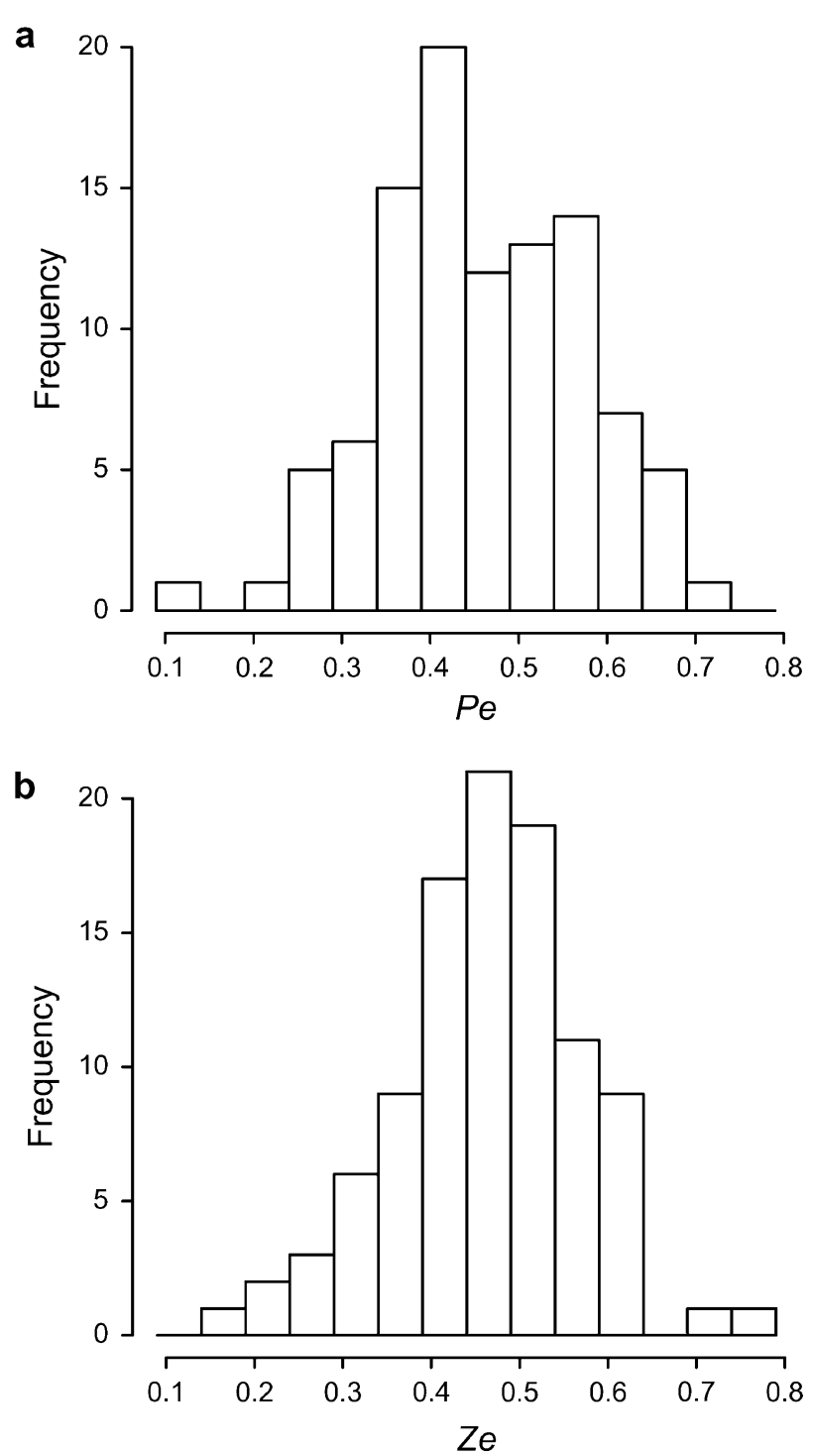

Figure 3. Histograms of Spearman rank correlations between true and posterior means of ram breeding values. $\mathrm{H} 4$ scenario for models $\mathrm{Pe}$ (a) and $\mathrm{Ze}$ (b).

the predicted values (posterior mean) was calculated for each combination of scenarios and models. Ze was best in all scenarios, but differences were small (Fig. 3). The ZIP model performed reasonably well in all scenarios, while pure Poisson models did well only in their own scenarios, with median correlations between 0.41 and 0.53 . 
Table V. Parameter estimates for the four scenarios by model.

\begin{tabular}{ccccccccccc}
\hline Model & $b_{0}$ & s.d. & $b_{\text {age }}$ & s.d. & $\sigma_{\text {ram }}^{2}$ & s.d. & $\sigma_{e}^{2}$ & s.d. & $\theta$ & s.d. \\
\hline $\mathrm{H} 1$ & -0.500 & & 0.520 & & 0.090 & & 0.000 & & 0.510 & \\
$\boldsymbol{Z}$ & $-\mathbf{0 . 5 0 3}$ & 0.097 & $\mathbf{0 . 5 2 1}$ & 0.017 & $\mathbf{0 . 1 0 4}$ & 0.042 & & & $\mathbf{0 . 5 0 4}$ & 0.022 \\
$Z e$ & $-\mathbf{0 . 5 2 5}$ & 0.102 & $\mathbf{0 . 5 2 3}$ & 0.018 & $\mathbf{0 . 1 0 2}$ & 0.042 & 0.022 & 0.010 & $\mathbf{0 . 5 0 0}$ & 0.023 \\
$P$ & -1.236 & 0.123 & $\mathbf{0 . 5 2 4}$ & 0.038 & $\mathbf{0 . 1 6 9}$ & 0.072 & & & & \\
$P e$ & -1.869 & 0.142 & $\mathbf{0 . 4 9 3}$ & 0.044 & $\mathbf{0 . 1 0 3}$ & 0.057 & 1.666 & 0.177 & & \\
& & & & & & & & & & \\
$\mathrm{H} 2$ & -0.550 & & 0.520 & & 0.090 & & 0.250 & & 0.480 & \\
$Z$ & -0.282 & 0.133 & $\mathbf{0 . 4 9 2}$ & 0.032 & $\mathbf{0 . 1 3 0}$ & 0.051 & & & $\mathbf{0 . 5 2 4}$ & 0.025 \\
$Z \boldsymbol{e}$ & $-\mathbf{0 . 5 2 6}$ & 0.139 & $\mathbf{0 . 5 1 5}$ & 0.028 & $\mathbf{0 . 1 0 1}$ & 0.046 & $\mathbf{0 . 2 5 1}$ & 0.058 & $\mathbf{0 . 4 8 2}$ & 0.028 \\
$P$ & -1.089 & 0.148 & $\mathbf{0 . 5 1 1}$ & 0.043 & $\mathbf{0 . 1 8 6}$ & 0.064 & & & & \\
$P e$ & -1.840 & 0.151 & $\mathbf{0 . 4 7 1}$ & 0.051 & $\mathbf{0 . 0 9 4}$ & 0.052 & 2.029 & 0.214 & & \\
& & & & & & & & & & \\
$\mathrm{H} 3$ & -2.500 & & 0.850 & & 0.090 & & 0.000 & & 0.000 & \\
$Z$ & $-\mathbf{2 . 4 7 7}$ & 0.102 & $\mathbf{0 . 8 4 5}$ & 0.017 & $\mathbf{0 . 1 0 2}$ & 0.041 & & & 0.014 & 0.006 \\
$Z e$ & $-\mathbf{2 . 4 8 5}$ & 0.108 & $\mathbf{0 . 8 4 5}$ & 0.017 & $\mathbf{0 . 0 9 9}$ & 0.040 & $\mathbf{0 . 0 1 8}$ & 0.009 & 0.014 & 0.005 \\
$\boldsymbol{P}$ & $\mathbf{- 2 . 4 9 7}$ & 0.104 & $\mathbf{0 . 8 4 8}$ & 0.017 & $\mathbf{0 . 1 0 2}$ & 0.039 & & & & \\
$P e$ & $-\mathbf{2 . 5 1 1}$ & 0.107 & $\mathbf{0 . 8 4 9}$ & 0.016 & $\mathbf{0 . 1 0 0}$ & 0.038 & 0.019 & 0.009 & & \\
& & & & & & & & & & \\
$\mathrm{H} 4$ & -2.500 & & 0.810 & & 0.090 & & 0.250 & & 0.000 & \\
$Z$ & $-\mathbf{2 . 2 6 8}$ & 0.347 & $\mathbf{0 . 7 9 3}$ & 0.053 & $\mathbf{0 . 1 4 0}$ & 0.052 & & & $\mathbf{0 . 0 6 3}$ & 0.087 \\
$Z e$ & $\mathbf{- 2 . 4 7 0}$ & 0.122 & $\mathbf{0 . 8 1 0}$ & 0.025 & $\mathbf{0 . 1 0 4}$ & 0.046 & $\mathbf{0 . 2 2 1}$ & 0.056 & 0.022 & 0.010 \\
$P$ & $\mathbf{- 2 . 3 9 7}$ & 0.137 & $\mathbf{0 . 8 1 0}$ & 0.030 & $\mathbf{0 . 1 4 3}$ & 0.053 & & & & \\
$\boldsymbol{P e}$ & $\mathbf{- 2 . 5 0 9}$ & 0.122 & $\mathbf{0 . 8 1 4}$ & 0.025 & $\mathbf{0 . 1 0 5}$ & 0.046 & $\mathbf{0 . 2 3 7}$ & 0.055 & & \\
\hline & & & & & & & & & & \\
\hline
\end{tabular}

The "true" value of each parameter is given in the line corresponding to each scenario (H1H4). Estimates for which the "true value" is inside a 2 standard deviations region are in boldface (see text for definition of parameters).

\subsection{Field data}

Given the simulation results, data were analysed using the two "best" models, $P e$ and $Z e$, including flock, year, flock $\times$ year and age effect as covariates, and ram as a genetic effect. The effects were removed successively from each main model and DIC was computed; full models were found to be the best. Since the effects of flock, year and flock-year interaction were negligible, these are not reported in Table VI. DIC $(p D)$ was 1103 (200.6) and 1070 (201.8) for the $Z e$ and $P e$ models, respectively. The number of spots increased with age, and there was no strong evidence of inflation at zero (the estimate of the probability of the perfect state, $\theta$, was 0.05 ). It seems that most of the overdispersion is due to unaccounted for between-individual variability. 
Table VI. Posterior median and quantiles $(2.5 \%$ and $97.5 \%)$ of the distribution of parameters, and difference in posterior predictive ability (DPPA) for $\mathrm{Pe}$ and $\mathrm{Ze}$ models applied to field data.

\begin{tabular}{crrrrrrr}
\hline \multirow{2}{*}{ Model } & \multicolumn{3}{c}{$Z e$} & & \multicolumn{3}{c}{$P e$} \\
\cline { 2 - 4 } & \multicolumn{1}{c}{$2.5 \%$} & Median & $97.5 \%$ & & $2.5 \%$ & Median & $97.5 \%$ \\
\hline$b_{0}$ & -2.490 & -1.692 & -0.926 & & -2.622 & -1.884 & -1.185 \\
$b_{\text {age }}$ & 0.465 & 0.611 & 0.761 & & 0.485 & 0.628 & 0.772 \\
$\sigma_{\text {ram }}^{2}$ & 0.008 & 0.092 & 0.474 & & 0.008 & 0.086 & 0.460 \\
$\sigma_{e}^{2}$ & 1.308 & 1.854 & 2.567 & & 1.513 & 2.026 & 2.736 \\
$\theta$ & 0.002 & 0.050 & 0.173 & & & \\
Heritability & 0.023 & 0.246 & 0.836 & & 0.016 & 0.166 & 0.714 \\
DPPA & -0.471 & -0.038 & 0.406 & & -0.500 & -0.040 & 0.423 \\
\hline
\end{tabular}

In standard animal breeding theory, in a sire model, heritability is defined as:

$$
h^{2}=\frac{4 \sigma_{\mathrm{ram}}^{2}}{\sigma_{\mathrm{ram}}^{2}+\sigma_{e}^{2}} .
$$

Estimates of heritability (in a log-scale) under the two models considered are displayed in Table VI. Posterior medians were 0.25 and 0.17 for the $Z e$ and $P e$ model, respectively, but the distribution was very skewed due to the few rams (19) used in the study.

\section{DISCUSSION}

While easier to measure than the number of dark fibres per animal, modelling the number of spots poses several challenges in regard to standard methodology of animal breeding. It is very difficult to obtain a good fit of the data with simple linear models with normal distributions for the random effects. Frequently used Box-Cox transformations (e.g., log or reciprocal) cannot be used, given the number of zeros. Additionally, there is the issue of an excess of zeros relative to Poisson sampling. One attractive model for dealing with this is the ZIP. One can think of a fraction $\theta$ of "perfect" animals that will never develop spots, whereas others only will develop spots at random, following a Poisson distribution with parameter $\lambda$. Moreover, variation in the $\lambda$ 's can be accounted for by a model including environmental and genetic factors, such as age, flock, year or ram.

We first compared the performance of four models (i.e., Poisson and ZIP with and without an error term in the regression) using a simulation that resembled the field data structure. Based on the end points considered, two "competitive" 
models emerged, $\mathrm{Pe}$ and $\mathrm{Ze}$, both including residuals. Fitting hierarchical models with an error term such as $P e$ and $Z e$ can be viewed as a log-normal mixture of Poisson and ZIP distributions, respectively [5]. This provides a very flexible structure, which explains at least partially, the good performance of these two models in the simulation results.

Using the DIC, the $P e$ model was best in most scenarios, in spite of a larger $p D$, a term that penalises the likelihood and that is associated with the effective number of parameters of the model. The results of the marginal likelihoods also supported this interpretation (Tab. IV), while the difference statistic for the posterior predictive ability (DPPA) displayed low discriminative power. However, the $P e$ model did not produce good estimates of the intercept (number of spots at age $=0$, i.e. birth) and of the residual variance, when data were generated from ZIP distributions (Tab. V). This suggests that even the results of DIC should be viewed with some caution. Furthermore, as pointed out by several discussants in Spiegelhalter et al. [30], the DIC may underpenalise model complexity. Several alternative versions of DIC were proposed by Celeux et al. [4] to address models with missing data or mixtures of distributions. However, despite important differences in performance, each alternative proposed has its own drawbacks and no single solution emerges as unanimously appropriate.

On the contrary, Ze was robust across all situations, since it estimated the true parameters well. A "stable model" is appealing under practical conditions. In animal breeding, a stable model with good predictive ability is desired. All models produced a good agreement between "true" and predicted breeding values, especially $Z e$, which maintained its ability across scenarios.

Based on the simulation, $P e$ and $Z e$ were chosen to analyse the field dataset. As expected, under the DIC Pe outperformed Ze. However, parameter estimates were similar (Tab. VI). This may be explained by the fact that the estimate of $\theta$ in $Z e$ was low, pointing to a relatively small effect of "perfect" individuals on inference when the Poisson model includes a residual.

A series of environmental and genetic factors may be related to the number of spots. Simple observation (even in humans) suggests that this number increases with age and environmental stress factors (e.g., solar irradiation can be invoked as causative agents [7,8,14,23]). Variability in the underlying genetic mechanisms responsible for the spots is likely, at least in different races, as well as in susceptibility to environmental stress factors.

The age of the animals was the main environmental factor to consider, consistently. However, it is not known if this relationship arises from an intrinsic ageing process independent of environmental factors, or if environmental stressors such as sun irradiation drive the process. Anyhow, it is possible to envisage management measures aiming to reduce incidence of dark fibres. If an intrinsic 
ageing process is the main factor, reducing the age at shearing could be a practice to take into consideration. This requires additional research.

In an animal breeding context, genetic and environmental variances are extremely important since they define heritability, a key parameter used to select among breeding strategies. The meaning of heritability in non-linear hierarchical models, such as $\mathrm{Pe}$ or $\mathrm{Ze}$, is not straightforward. However, the magnitude of heritability suggests scope for genetic selection. Different simulations indicated that predicted breeding values (for $\log \lambda$ ) were in good agreement with "true" values, so these models are probably useful for selection purposes.

In summary, hierarchical models for count data were studied with the aim of defining strategies for reducing incidence of dark fibres in wool from Corriedale sheep. ZIP and Poisson models with random residuals performed better than their counterparts without residuals. Ageing related processes seem to drive the number of dark spots in sheep, and further research should be done to address this underlying phenomenon.

\section{ACKNOWLEDGEMENTS}

We would like to thank the Associate Editor and two anonymous reviewers for their useful comments and suggestions, and Gonzalo I. Pereira, Carlos R. Lopez, Lucía Surraco and Fabián Gonzalez for field data collection. We are indebted to Gustavo de los Campos, Guilherme J.M. Rosa, Agustín Blasco and Martín Graña for helpful discussions. This work was partially supported by Comisión Sectorial de Investigación Científica - Uruguay, and by grant PDT35-02 from Programa de Desarrollo Tecnológico (Uruguay). Support from the Wisconsin Agriculture Experiment Station, and from grant NSF DMS-NSF DMS-044371 is also acknowledged.

\section{REFERENCES}

[1] Cardellino R., Herencia de fibras coloreadas, Produc. Ovina 6 (1994) 19-37.

[2] Cardellino R., Mendoza J., Fibras coloreadas en tops con lanas acondicionadas (zafra 94-95), Rev. Lananoticias SUL 115 (1996) 37-40.

[3] Cardellino R., Guillamón B.E., Severi J.F., Origen de las fibras coloreadas en tops de lana uruguaya, Produc. Ovina 3 (1990) 81-83.

[4] Celeux G., Forbes F., Robert C.P., Titterington D.M., Deviance information criteria for missing data models, Bayesian Anal. 1 (2006) 651-674.

[5] Draper D., Tutorial 1: Hierarchical Bayesian Modeling, in: 6th World Meeting International Society for Bayesian Analysis, 28 May-1 June, 2000, Hersonissos, Heraklion, Crete. 
[6] Enns R.M., Nicoll G.B., Incidence and heritability of black wool spots in Romney sheep, N. Z. J. Agric. Res. 45 (2002) 67-70.

[7] Fears T.R., Scotto J., Schneiderman M.A., Skin cancer, melanoma, and sunlight, Am. J. Public Health 66 (1976) 461-464.

[8] Fears T.R., Scotto J., Schneiderman M.A., Mathematical models of age and ultraviolet effects on the incidence of skin cancer among whites in the United States, Am. J. Epidemiol. 105 (1977) 420-427.

[9] Fleet M., Pigmentation types. Understanding the heritability and importance, Wool Tech. Sheep Breed. 44 (1996) 264-280.

[10] Fleet M.R., Forrest J.W., The occurrence of pigmented skin and pigmented wool fibres in adult Merino sheep, Wool Tech. Sheep Breed. 33 (1984) 83-90.

[11] Fleet M., Lush B., Sire effects on visible pigmentation in a Corriedale flock, Wool Tech. Sheep Breed. 45 (1997) 167-173.

[12] Fleet M.R., Ponzoni R.W., Fibras pigmentadas en vellones blancos, in: Larrosa J.R., Bonifacino L.A. (Eds.), Seminario Científico Técnico Regional de Lanas, Montevideo, 1985, pp. 135-142.

[13] Fleet M.R., Stafford J.E., The association between non-fleece pigmentation and fleece pigmentation in Corriedale sheep, Anim. Prod. 49 (1989) 241-247.

[14] Forrest J.W., Fleet M.R., Pigmented spots in the wool-bearing skin induced by ultraviolet light, Aust. J. Biol. Sci. 39 (1986) 123-136.

[15] Gianola D., Statistics in animal breeding: angels and demons, in: Proceedings of the 8th World Congress on Genetics Applied to Livestock Production, 13-18 August, 2006, CD Paper 00-03, Belo Horizonte-MG, Brazil, 8 p.

[16] Kremer R., Urioste J.I., Naya H., Rosés L., Rista L., López C., Incidence of skin spots and pigmentation in Corriedale sheep, in: IX World Conference on Animal Production, 26-31 October, 2003, Porto Alegre, Brazil.

[17] Lambert D., Zero-inflated Poisson regression, with an application to defects in manufacturing, Technometrics 34 (1992) 1-14.

[18] Larrosa J.R., Orlando D., Incidencia de fibras oscuras en lanas peinadas uruguayas, in: An. Fac. Vet. Uruguay, Montevideo, 21/25, 1984-1988, pp. 71-78.

[19] Martínez-Ávila J.C., Spangler M., Rekaya R., Hierarchical model for zero-inflated count data: a simulation study, in: Proceedings of the 8th World Congress on Genetics Applied to Livestock Production, 13-18 August, 2006, CD Paper 24-08, Belo Horizonte-MG, Brazil, 4 p.

[20] Mendoza J., Cardellino R., Maggiolo J., Garín M., Fibras coloreadas en Corriedale, Lananoticias 129 (2001) 37-40.

[21] McCullagh P., Nelder J.A., Generalized Linear Models, Chapman and Hall, London, 1989.

[22] Newton M.A., Raftery A.E., Approximate Bayesian inference by the weighted likelihood bootstrap (with discussion), J. Roy. Stat. Soc. B 56 (1994) 1-48.

[23] Oliveria S.A., Saraiya M., Geller A.C., Heneghan M.K., Jorgensen C., Sun exposure and risk of melanoma, Arch. Dis. Child. 91 (2006) 131-138. 
[24] Pereira G.I., Miquelerena J.M., Urioste J.I., Naya H., Kremer R., Lopez A., Surraco L., Presencia de fibras pigmentadas en una majada experimental, Corrie- dale, in: 12th Corriedale World Congress, 1-10 September, 2003, Montevideo, Uruguay, p. 109.

[25] Pinheiro J.C., Bates D.M., Mixed-effects Models in S and S-PLUS, Springer, New York, 2000.

[26] R Development Core Team, R: A Language and Environment for Statistical Computing, 2007, R Foundation for Statistical Computing, Vienna, Austria, ISBN 3-900051-07-0, http://www.R-project.org.

[27] Rodrigues-Motta M., Gianola D., Heringstad B., Rosa G.J.M., Chang Y.M., A zero-inflated Poisson model for genetic analysis of number of mastitis cases in Norwegian Red cows, J. Dairy Sci. 90 (2007) 5306-5315.

[28] Sorensen D., Gianola D., Likelihood, Bayesian, and MCMC Methods in Quantitative Genetics, Springer-Verlag, New York, 2002.

[29] Sorensen D., Waagepetersen R., Normal linear models with genetically structured residual variance heterogeneity: a case study, Genet. Res. Camb. 82 (2003) 207-222.

[30] Spiegelhalter D.J., Best N.G., Carlin B.P., van der Linde A., Bayesian measures of model complexity and fit (with discussion), J. Roy. Stat. Soc. B 64 (2002) 583-639.

[31] Thomas A., Hara B.O., Ligges U., Sturtz S., Making BUGS Open, R News 6 (2006) 12-17. 